\title{
Pemphigoid gestationis: current perspectives
}

This article was published in the following Dove Press journal:

Clinical, Cosmetic and Investigational Dermatology

8 November 2017

Number of times this article has been viewed

\section{Christine Sävervall' \\ Freja Lærke Sand' Simon Francis Thomsen ${ }^{1,2}$ \\ 'Department of Dermatology, Bispebjerg Hospital, Copenhagen, Denmark; ${ }^{2}$ Department of Biomedical Sciences, University of Copenhagen, Copenhagen, Denmark}

Abstract: Many skin diseases can occur in pregnant women. However, a few pruritic dermatological conditions are unique to pregnancy, including pemphigoid gestationis (PG). As PG is associated with severe morbidity for pregnant women and carries fetal risks, it is important for the clinician to quickly recognize this disease and refer it for dermatological evaluation and treatment. Herein, we review the pathogenesis, clinical characteristics, and management of PG. Keywords: pemphigoid gestationis, pregnancy, skin diseases

\section{Introduction}

Skin changes are common during pregnancy and can be divided into benign physiologic skin changes due to normal hormonal/physiological changes, ${ }^{1}$ alterations in preexisting skin diseases because of immunohormonal changes, and pregnancy-specific dermatoses. Pregnancy-specific dermatoses represent a group of skin diseases that occur only during pregnancy and/or the immediate postpartum period. Severe pruritus represents the leading symptom commonly followed by a more widespread skin rash. Pregnancy-specific dermatoses include: pemphigoid gestationis (PG), polymorphic eruption of pregnancy (PEP), intrahepatic cholestasis of pregnancy (ICP), and atopic eruption of pregnancy (AEP). ${ }^{2}$

PG or gestational pemphigoid is a rare pregnancy-associated autoimmune skin disorder that is immunologically and clinically similar to the pemphigoid group of autoimmune blistering skin disorders. The pathogenesis is not yet fully established, but it belongs to the group of autoimmune skin disorders characterized by an immune response directed against different hemidesmosomal proteins affecting the adherence between the dermis and epidermis causing blistering of the skin and mucosal membranes. ${ }^{3}$ Clinically, $\mathrm{PG}$ is characterized by intense pruritus and polymorphic skin eruptions. Pruritus can emerge before skin lesions and remain the only symptom. In more severe cases, skin lesions develop including erythematous patches and plaques, sometimes followed by urticarial rash and blisters. PG was previously termed herpes gestationis because the morphology of the blisters was similar to that of herpes, but it was shown not to be related to, or associated with, any prior or active herpes virus infection.

Some pregnancy-specific dermatoses carry considerable morbidity for pregnant women and can pose a significant risk to the fetus, such as premature birth and small-for-gestational age babies. ${ }^{4}$ It is therefore important to recognize and separate
Correspondence: Simon Francis Thomsen Department of Dermatology, Bispebjerg Hospital, Bispebjerg Bakke 23, DK-2400

Copenhagen NV, Denmark

Tel +4538635844

Fax +45 38639785

Email simonfrancisthomsen@gmail.com 
these diseases from other benign pregnancy-associated skin changes. Pregnant women who present with a pruritic rash should always get immediate clinical evaluation, and if necessary referred to a dermatologist. In this review, we elucidate the epidemiology, pathogenesis, clinical characteristics, treatment, and complications of PG.

\section{Epidemiology and risk factors}

General population studies on the epidemiology of PG are rare. Population-based studies have reported an annual incidence ranging between 0.5 and 2.0 cases per 1 million people in France, Kuwait, Iran, and Germany. ${ }^{5-8}$ The incidence is estimated to be approximately 1 in 60,000 pregnancies..$^{9,10}$ The disease shows a worldwide distribution ${ }^{2,5,6,8,11,12}$ and no differences in ethnicity. ${ }^{13}$ The median age of affected women varies between 17 and 41 years, with a median age of onset around 26-32 years. ${ }^{11,14-16}$

PG mainly affects multiparous women in their second or third trimester of pregnancy, but onset in the first trimester or postpartum period is also reported..$^{2,11,12,14,16-19}$ In a case series from the UK of women with PG including 117 pregnancies, the time of onset of $\mathrm{PG}$ ranged from 5 weeks of gestation to 35 days postpartum. Of the 117 pregnancies, 21 (17.9\%) presented in the first trimester, $40(34.2 \%)$ presented in the second trimester, and $40(14.2 \%)$ presented in the third trimester, whereas in $16(13.7 \%)$ the eruption began postpartum. ${ }^{14}$ In another case series from Austria including 21 pregnancies, $15(71 \%)$ had onset during the third trimester and $6(29 \%)$ had an onset in the second trimester. ${ }^{2}$ The tendency for onset late in pregnancy was also confirmed in a third study from Saudi Arabia including 32 patients which showed that $84 \%$ of women had onset of PG during the second or third trimester. ${ }^{11}$ Finally, in a smaller study from the UK including 15 cases of PG, 8 women had onset in the third trimester, 2 in the immediate postpartum period, whereas 4 presented in the second trimester. ${ }^{19}$

Recurrences in subsequent pregnancies are common and are usually more severe and with an earlier onset. ${ }^{1,15,17,20}$ Studies show recurrences in $33 \%-50 \%$ of patients, ${ }^{11,21}$ but cases of uninvolved or skipped pregnancies following a previously affected pregnancy are also reported. In a case study including 25 patients with $\mathrm{PG}, 2$ patients (8\%) presented with an uninvolved pregnancy following a previously affected pregnancy. ${ }^{22}$ In another study of 87 patients with PG, 7 patients $(8 \%)$ had an uninvolved pregnancy. This study also showed that skipped pregnancies could not be explained by a change in sexual partner or by the mother and the fetus being fully compatible at the DR locus. ${ }^{14}$ Why skipped pregnancies occur is still unknown. Both primiparous and multiparous women are affected, but most cases are reported to occur in multiparous women, ${ }^{11,14,19}$ although this is not congruent with a study of 23 cases where $56.5 \%$ of the women with PG were primiparous. ${ }^{15}$ There is no association between change in partner and development of $\mathrm{PG},{ }^{14}$ which was previously suggested. ${ }^{22}$

\section{Pathogenesis}

The pathogenesis of PG is considered to be similar to that of bullous pemphigoid, which is characterized by deposition of autoreactive antibodies directed against two hemidesmosomal proteins, BP180 and BP230, within the dermoepidermal junction, resulting in the formation of bullae and skin erosions. ${ }^{23}$ In PG, the first immune response occurs within the placenta. Placental trophoblasts and amniochorionic stromal cells show an abnormal expression of major histocompatibility complex (MHC) class II antigens ${ }^{24}$ allowing the presentation of BP180 (also known as BPAG1 or collagen XVII) protein to the maternal immune system. BP180 is a key structural protein of hemidesmosomes linking the epidermis and dermis. ${ }^{25} \mathrm{BP} 180$ is found in placental tissue, in fetal membranes, and also in the basement membrane zone of the skin. These specific proteins presented in the placenta are recognized as foreign, causing subsequent production of anti-placental IgG antibodies that cross-react with the same BP180 proteins in the skin. The binding of these antibodies to the basement membrane of the skin triggers an autoimmune response that consists of complement activation, deposition of immunecomplexes, consecutive chemoattraction of eosinophil granulocytes, and subsequent degranulation, resulting in tissue damage and blister formation. ${ }^{26}$

Immunogenesis is also found to play a role in the pathogenesis of PG. It has been delineated that PG has a strong association with maternal MHC class II antigens haplotypes HLA-DR3 and HLA-DR4. ${ }^{27}$ These haplotypes have been shown to be more common in women with PG compared to a healthy control population. The presence of MHC II-class HLA-antigens DR3 was found in 61\%-80\% of patients compared to only $22 \%$ in controls. DR4 was found in $52 \%-53 \%$ of patients compared to $33 \%$ in controls. Notably, the combination of these two haplotypes was found in $45 \%$ of patients compared to $3 \%$ of controls. ${ }^{27,28}$

Fluctuations in sex hormones are also proposed to play a role in the pathogenesis of PG. This is due to observations of a clinical flare or exacerbation during menstruation or following administration of oral contraceptives postpartum, ${ }^{9,14,29,30}$ but these observations are not consistent across studies. ${ }^{14}$ 


\section{Clinical features}

PG initially presents with intense pruritus and inflammatory skin lesions (Table 1). Pruritus can remain the only symptom, but mostly it develops into eruptive polymorphic skin lesions. ${ }^{2,15,16}$ Eruptive skin lesions initially present as urticarial papules and annular plaques, followed by vesicles and finally large tense bullae on an erythematous background (Figure 1). Skin lesions typically develop on the abdomen, characteristically involving the umbilical region (Figures 2 and 3)..$^{2,11,14,19}$ In $90 \%$ of the cases, it later spreads to the rest of the abdomen (Figure 4), and in some patients the involvement of thighs (Figure 5), palms, and soles can be prominent. ${ }^{29} \mathrm{In}$ a study of 23 patients, extremities were the most common site of involvement $(100 \%) \cdot{ }^{15}$ The mucous membranes and face are usually spared. ${ }^{18,31}$

Many patients experience remission during late pregnancy, sometimes followed by a flare immediately after delivery. The flare usually settles over a period of 4 weeks without

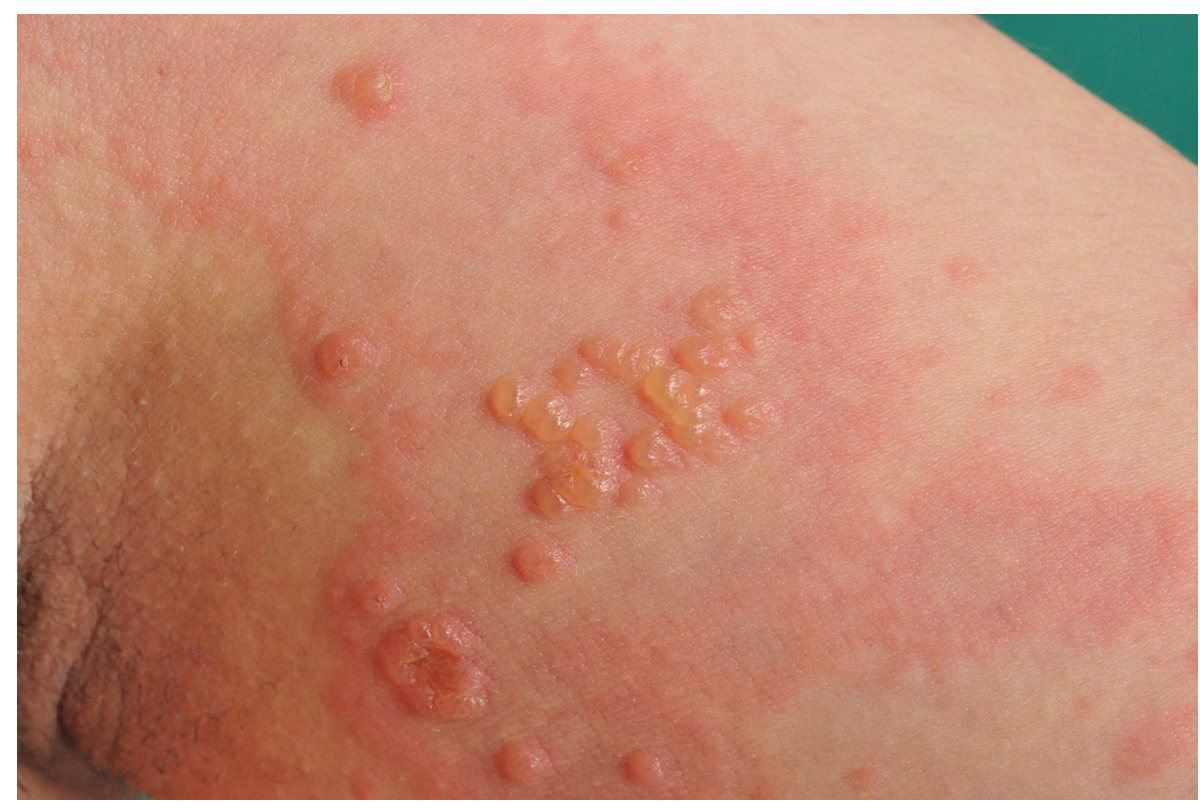

Figure I Blister formation in pemhigoid gestationis.

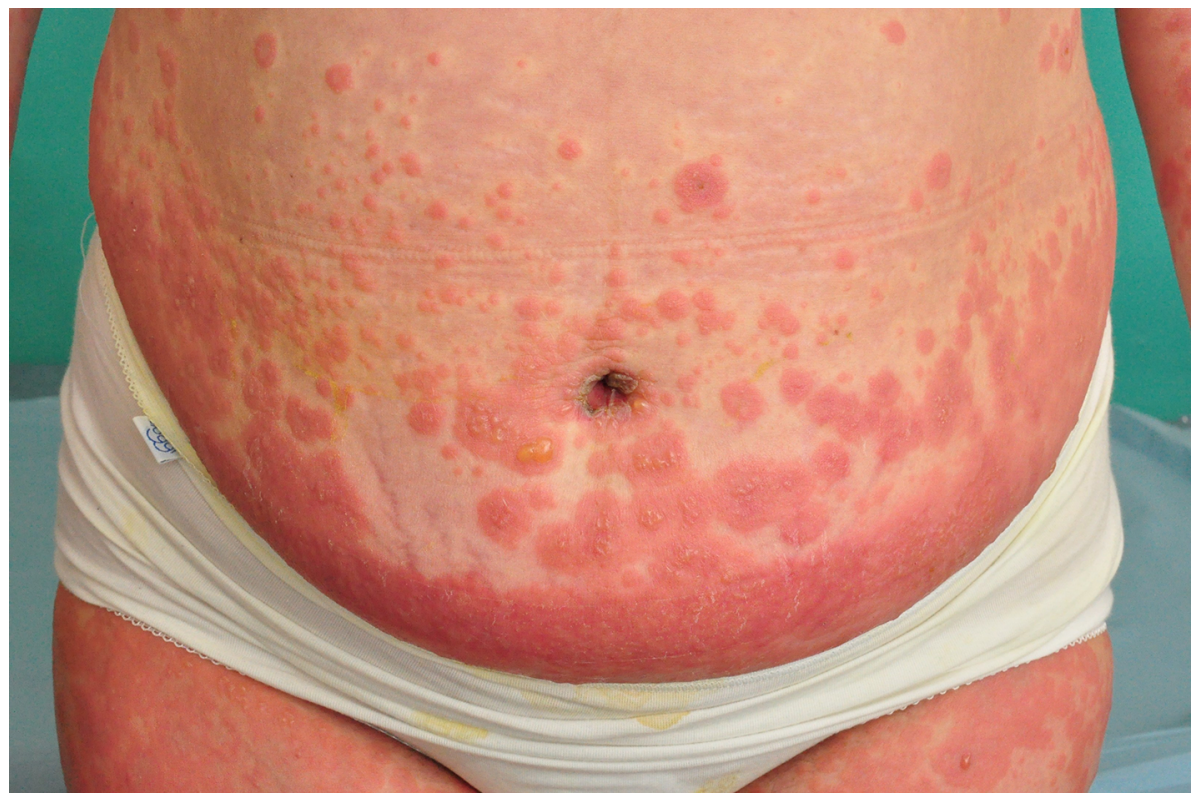

Figure 2 Umbilical involvement in pemhigoid gestationis. 


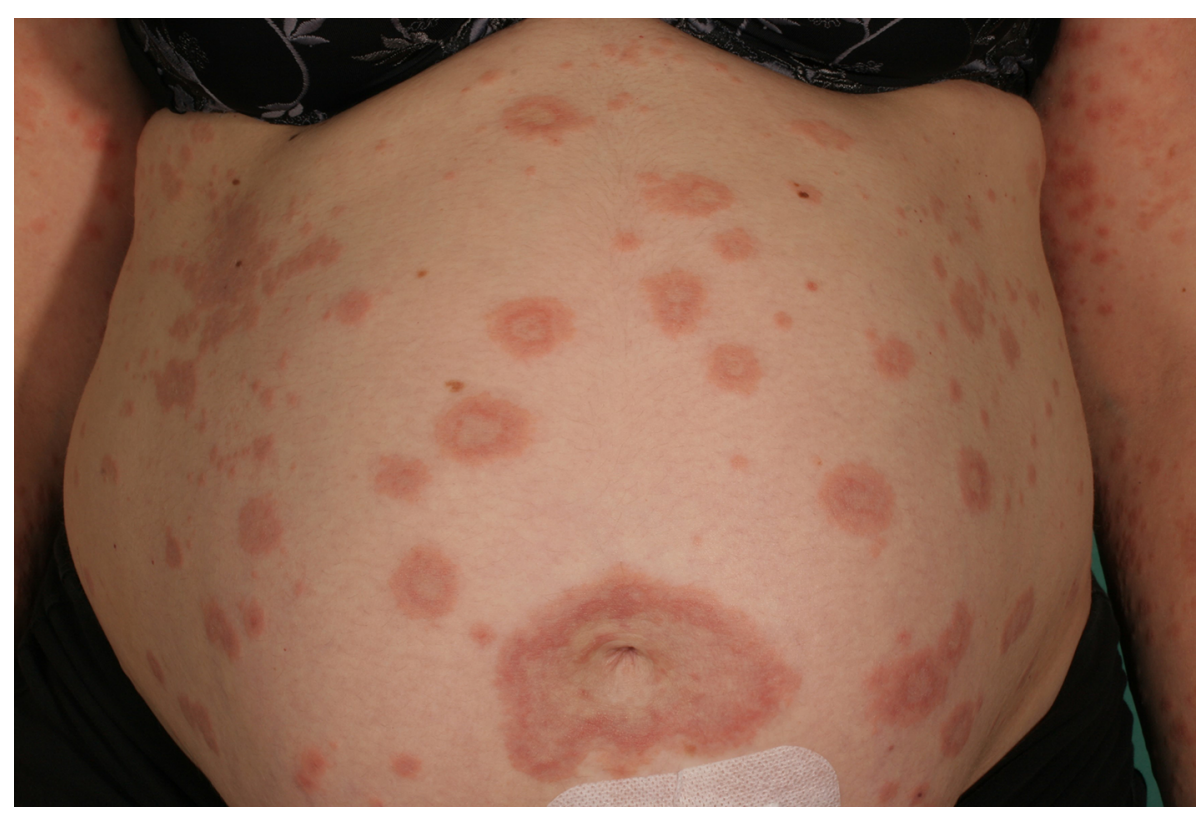

Figure 3 Erythemtous, annular patches in pemhigoid gestationis.

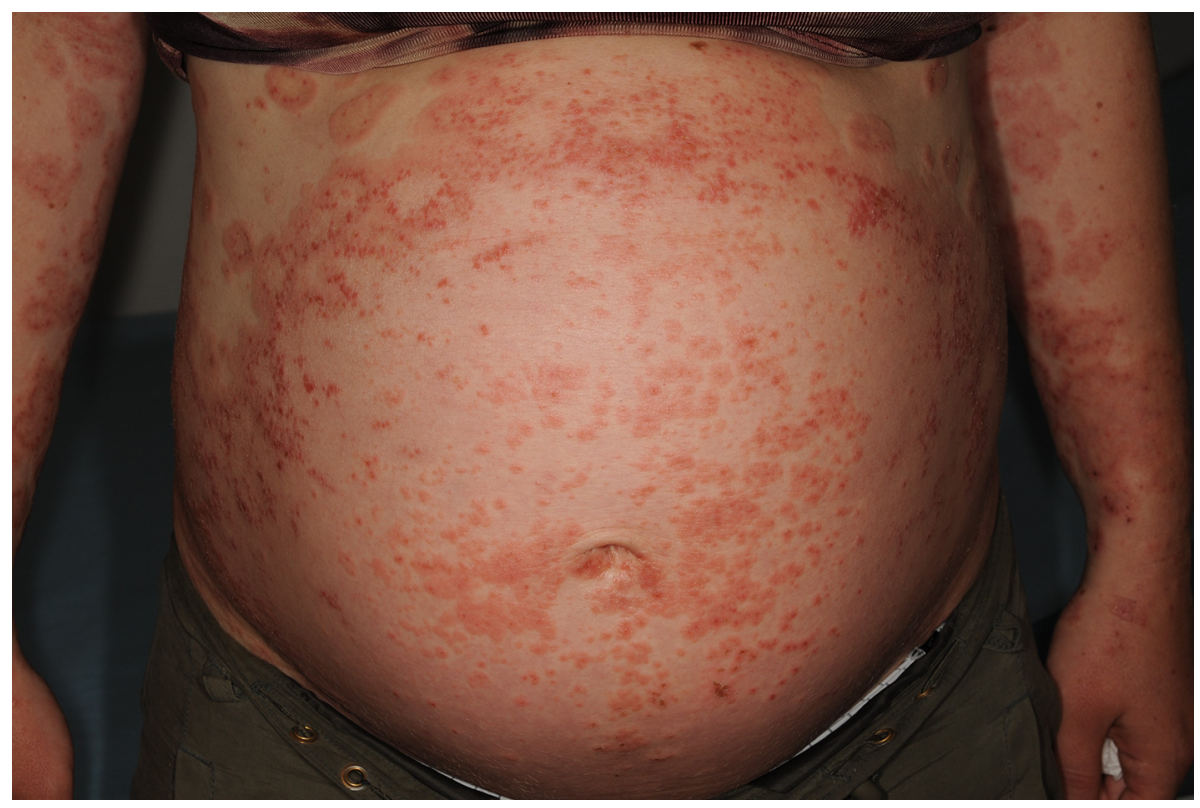

Figure 4 Papular eruption in pemhigoid gestationis.

recurrence. ${ }^{19}$ This supports the plausible pathogenic role of fluctuations in sex hormones. The disease is self-limiting, and after delivery the lesions usually resolve spontaneously within weeks to months. Rarely, a severe course with persistence of skin lesions over several years is seen. ${ }^{1,14}$ In a study of 87 patients from the UK, the duration of active disease ranged from 2 weeks postpartum to 12 years postpartum. The majority of patients were symptom free after 6 months, with a mean disease duration of 28.4 weeks and a median duration of 16 weeks. ${ }^{14}$ Conversion from PG to BP should be considered in extremely persistent cases. ${ }^{32}$ Some patients experience a flare with the use of oral contraceptives; 6 (10.7\%) out of 56 patients showed a flare with the use of oral contraceptives postpartum ${ }^{14}$ compared to previous studies suggesting a risk of exacerbation of $50 \%{ }^{9}$ 


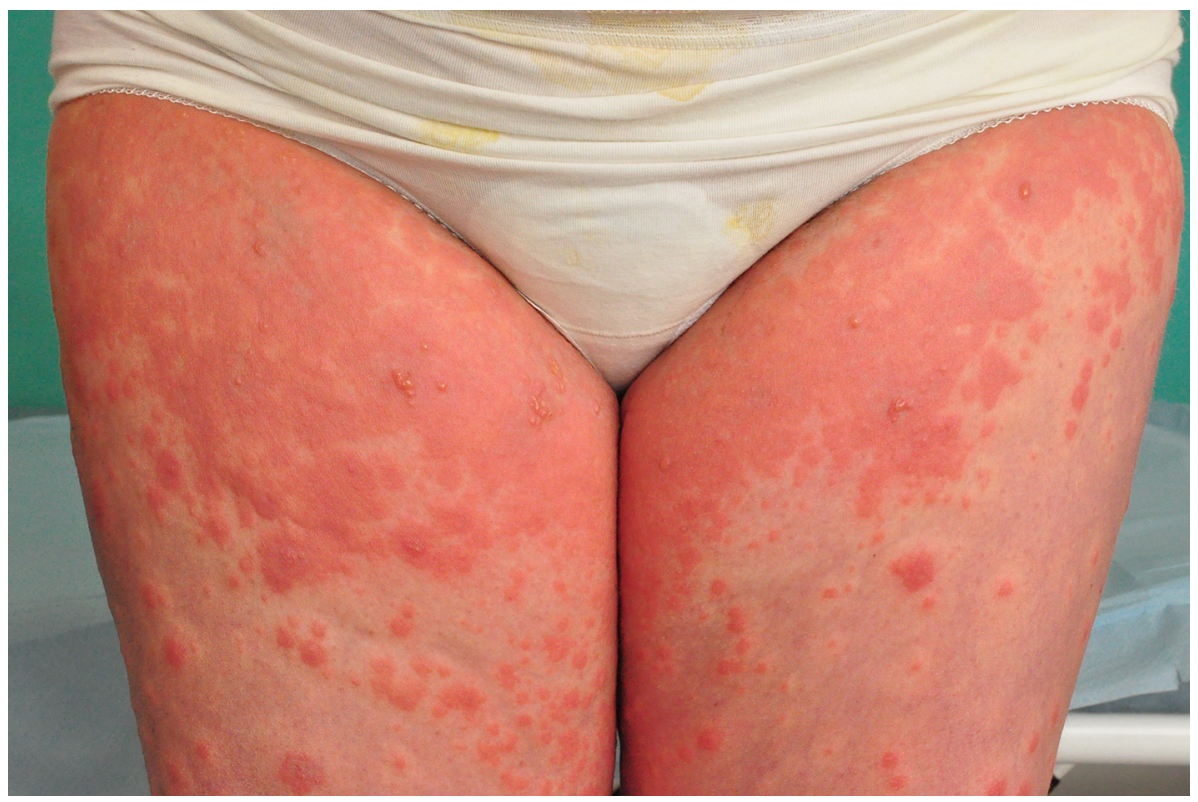

Figure 5 Urticaria-like eruption in pemhigoid gestationis.

\section{Diagnosis}

There are several ways to identify PG: clinical evaluation, histological findings, direct immunofluorescence (DIF) or indirect immunofluorescence (IIF), enzyme-linked immunosorbent assay (ELISA), and C4d immunochemistry can all be used to diagnose PG. C4d immunochemistry is not standard of care and still considered experimental. DIF is the most frequently used method to detect $\mathrm{PG}$, but recently other tests have been suggested to replace this method.

The histopathology of PG varies with the severity and the stage of the disease. In the early pre-bullous stage the classic histopathologic findings are urticarial lesions characterized by edema of the upper and mid-dermis with a perivascular infiltrate of lymphocytes, histiocytes, and eosinophils. In the later, bullous stage subepidermal split formations and bullae become evident. ${ }^{1}$ These histopathologic findings are not specific for PG and can also be seen in PEP. DIF demonstrates a linear deposition of $\mathrm{C} 3$ (complement 3 ) and $\mathrm{IgG}$ autoantibodies at the dermoepidermal junction. ${ }^{18,29} \mathrm{C} 3$ is reported in $100 \%$ of cases, while IgG is seen in $25 \%-50 \%$ of cases. ${ }^{29}$ To avoid a skin biopsy, circulating autoantibodies can be detected using complement-binding tests, such as IIF or ELISA. IIF detects IgG autoantibodies targeting the basement membrane of the skin in $30 \%-100 \%$ of cases. ${ }^{2}$ ELISA would typically reveal circulating IgG antibodies against BP180, particularly against the NC16A domain of BP180. This test has shown a specificity of $94 \%-98 \%$ and a sensitivity of $86 \%-97 \%$ in the detection of BP180 antibodies in patients with PG. ${ }^{33-35}$ ELISA is suitable to monitor disease activity because serum levels of anti-BP180 NC16A correlate with disease severity. ${ }^{33,34}$ In a more recent study, routine immunohistochemistry showed a linear $\mathrm{C} 4 \mathrm{~d}$ immunoreactant deposition along the basement membrane in $100 \%$ of the patients with PG compared to $0 \%$ in patients with PEP. ${ }^{36}$ This method can be used to separate PG from the other pregnancy-specific dermatoses such as PEP, ICP, and AEP or other skin diseases.

\section{Differential diagnoses}

There are several skin disorders with similar symptoms and clinical presentation as the pregnancy-specific dermatoses that may present during pregnancy. Pruritus is the leading symptom in the pregnancy-specific dermatoses, but pruritus also occurs in more common diseases such as urticaria, drug hypersensitivity reactions, contact dermatitis and other eczemas, pityriasis rosea, pityriasis versicolor, and yeast folliculitis as well as other types of folliculitis, miliaria, and scabies, which should be considered as differential diagnoses and excluded before extended investigations are initiated. ${ }^{37}$ The most important differential diagnoses for PG are the other pregnancy-specific dermatoses, which include AEP, PEP, and ICP. AEP is the most common pregnancy-specific dermatosis. It can be differentiated from PG by the time of onset. AEP presents early in the first or second trimester, whereas PG most commonly is seen during the second or third trimester. The most difficult disease to separate from PG is PEP. The symptoms are similar and the routine 
Table I Overview of PG

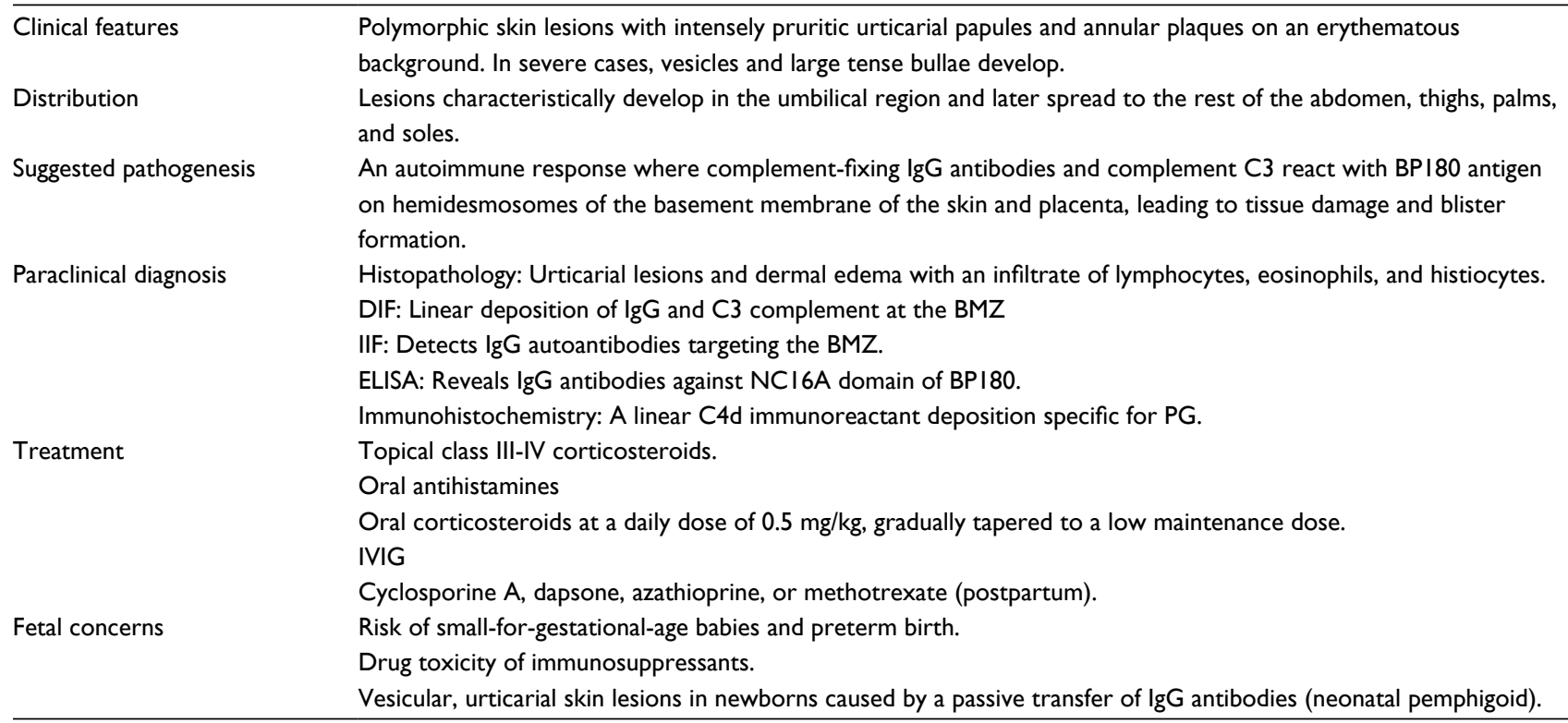

Abbreviations: BMZ, basement membrane zone; DIF, direct immunofluorescence; ELISA, enzyme-linked immunosorbent assay; IIF, indirect immunofluorescence; IVIG, intravenous immunoglobulin; PG, pemphigoid gestationis.

histopathologic findings are indistinguishable, but in PEP the eruption often shows sparing of the umbilical region unlike PG, which often starts in the umbilicus. To differentiate PG from PEP, additional paraclinical tests such as DIF and skin biopsy are required. ${ }^{38}$ ICP presents with pruritus, and it has an onset during late pregnancy and is associated with fetal risks. In ICP, skin lesions are secondary to scratching, and jaundice may occur due to elevated levels of total serum bile acid, which separates it from PG.

\section{Treatment}

The aim of the treatment is to reduce pruritus and to prevent the advancement of new blisters. The treatment strategy depends on the severity of the disease. In mild cases, the use of class III or IV topical corticosteroids is sufficient. In more severe cases, oral corticosteroids are necessary. ${ }^{14}$ Preferred corticosteroids are prednisone and prednisolone. They are nonflourinated glucocorticoids that are inactivated by the 11 - $\beta$-hydroxylase enzyme of the placenta, resulting in a lowering of the steroid concentration crossing the placenta. ${ }^{39}$ Minimum effective doses should be used to reduce the risk of side effects, starting with a daily dose of $0.5 \mathrm{mg} / \mathrm{kg}$ (or less), gradually tapered to a lower maintenance dose. If exacerbation in the peripartum period occurs, the maintenance dose can be increased. The duration of treatment postpartum is individualized, but since the majority of patients are symptom free after 6 months, it should be planned as part of the therapeutic taper. In addition to corticosteroids, oral antihistamines can be used to control pruritus. Ultraviolet light therapy is relatively contraindicated as it may promote new blister formation. In unresponsive cases, patients may benefit from systemic immunoadsorption, a blood-purification technique that enables the selective removal of immunoglobulins from separated plasma through high-affinity adsorbers, and intravenous immunoglobulin (IVIG) ${ }^{40-44}$ Immunoadsorption is rare and not available in all countries. IVIG is a useful treatment because of its availability and a good safety profile for the mother and the fetus, and this is therefore more standard of care. In case of persisting (postnatal) symptoms, systemic immunosuppressants such as cyclosporine A, dapsone, azathioprine, or methotrexate might be beneficial. ${ }^{30}$ The use of topical steroids, regardless of potency, shows no significant increase in adverse pregnancy outcome.$^{45}$ Low birth weight has been associated with the use of very potent topical steroids in one study of poor methodological quality. ${ }^{45} \mathrm{Common}$ side effects with the use of systemic immunosuppressants are nausea and loss of appetite, but there is no evidence of an increased risk of adverse pregnancy outcome. ${ }^{46}$ Use of cyclosporine A is associated with multiple side effects such as high blood pressure, renal insufficiency, bone marrow suppression, increased hair growth, headache, and cancer. If used during pregnancy, it can cause preterm birth but is considered not to be associated with birth defects. Dapsone is available both for topical and oral use. Severe side effects 
of oral dapsone include hemolysis and liver inflammation. Dapsone should not be used during pregnancy because of insufficient data on side effects in pregnant women. Azathioprine may cause bone marrow suppression, liver impairment, and hypersensitivity reactions. Pregnant women should be carefully monitored if treated with azathioprine because of a small risk of birth defects. Methotrexate may cause elevated liver enzymes and bone marrow suppression. It should not be used during pregnancy or during breast-feeding because of a high teratogenic risk, embryotoxicity, and spontaneous abortion. In a study from the UK of 87 patients 13 (18.8\%) out of 69 were treated with topical corticosteroids without systemic therapy, and $56(81.2 \%)$ out of 69 required systemic corticosteroids with initial doses of prednisolone in the range of 5-110 $\mathrm{mg}$ daily. Topical corticosteroids were inadequate once the vesico-bullous eruptions had developed. Most patients experienced remission with the use of systemic corticosteroids, but $15(21.7 \%)$ required additional treatment with other systemic immunosuppressants. Two patients were unresponsive to treatment and eruptions persisted for more than 10 years. ${ }^{14}$ In a study from Saudi Arabia of 32 patients, $75 \%$ responded well to oral corticosteroids. One patient needed IVIG. The vast majority of the patients $(61 \%)$ became free of symptoms within 1-2 months of treatment. ${ }^{11}$ A study from the UK of 15 patients showed that eight women were treated with systemic corticosteroids with starting doses of prednisolone between 30 and $40 \mathrm{mg} / \mathrm{d}$. The remaining women were treated with potent topical corticosteroids. Two women required additional immunosuppressant therapy for recalcitrant disease. ${ }^{19}$

\section{Fetal concerns}

Fetal prognosis is generally good, but PG is associated with fetal risks such as small-for-gestational-age babies and premature birth. ${ }^{47}$ Onset in the first or second trimester and the presence of blisters are found to be related to adverse pregnancy outcomes. ${ }^{4}$ Due to passive transfer of antibodies from the mother to the fetus, about $10 \%$ of newborns may develop mild urticaria-like or vesicular skin lesions (neonatal pemphigoid). ${ }^{28}$ The lesions are self-limiting, as within days to weeks antibody levels decrease.

Systemic use of corticosteroids does not appear to affect the fetal outcome. ${ }^{4}$ Methotrexate is toxic and contraindicated during pregnancy. Azathioprine and cyclosporine A can be used during pregnancy; however, drug toxicity in the mother related to medical treatment should be monitored closely because of an increased risk of birth defects and preterm birth.

\section{Comorbidities}

PG is associated with the autoimmune Graves' (hyperthyroidism) disease. ${ }^{14,48}$ In a study of 87 patients, the incidence of Graves' disease in PG was significantly increased to $10.3 \%$ compared to $0.4 \%$ in the normal population. ${ }^{14}$ This can partially be explained by the presence of HLA-DR3 and DR4.

\section{Conclusion}

PG is a rare autoimmune blistering skin disorder associated exclusively with pregnancy. Pathophysiologically, it is similar to bullous pemphigoid seen in elderly patients. The clinical presentation is characterized by intense pruritus and polymorphic skin lesions including blisters. The diagnosis is based upon clinical presentation and typical histopathological and laboratory findings. PG is self-limiting, but symptoms can be reduced with the use of topical and systemic corticosteroids, oral antihistamines, and systemic immunosuppressants. The prognosis is good, but PG is associated with fetal risks such as small-for-gestational-age babies and premature birth. Patients with PG should therefore be informed about the natural course of the disease and treatment possibilities. In addition, they should be informed about fetal prognosis, the possibility of relapse after delivery, relapse with the use of hormonal contraceptives, and the risk of relapse in subsequent pregnancies. Referral to dermatological evaluation is essential.

\section{Consent}

Written informed consent has been obtained from all patients for the publication of their images.

\section{Disclosure}

The authors report no conflicts of interest in this work.

\section{References}

1. Ambros-Rudolph CM. Dermatoses of pregnancy - clues to diagnosis, fetal risk and therapy. Ann Dermatol. 2011;23(3):265-275.

2. Ambros-Rudolph CM, Mullegger RR, Vaughan-Jones SA, Kerl H, Black MM. The specific dermatoses of pregnancy revisited and reclassified: results of a retrospective two-center study on 505 pregnant patients. J Am Acad Dermatol. 2006;54(3):395-404.

3. Enno Schmidt M, Zillikens D. Pemphigoid diseases. Lancet. 2013;381(9863):320-332.

4. Chi CC, Wang SH, Charles-Holmes R, et al. Pemphigoid gestationis: early onset and blister formation are associated with adverse pregnancy outcomes. Br J Dermatol. 2009;160(6):1222-1228.

5. Nanda A, Dvorak R, Al-Saeed K, Al-Sabah H, Alsaleh QA. Spectrum of autoimmune bullous diseases in Kuwait. Int $J$ Dermatol. 2004;43(12):876-881.

6. Bertram F, Bröcker EB, Zillikens D, Schmidt E. Prospective analysis of the incidence of autoimmune bullous disorders in Lower Franconia, Germany. J Dtsch Dermatol Ges. 2009;7(5):434-440. 
7. Bernard P, Vaillant L, Labeille B, et al. Incidence and distribution of subepidermal autoimmune bullous skin diseases in three French regions. Bullous Diseases French Study Group. Arch Dermatol. 1995;131(1):48-52.

8. Daneshpazhooh M, Chams-Davatchi C, Payandemehr P, Nassiri S, Valikhani M, Safai-Naraghi Z. Spectrum of autoimmune bullous diseases in Iran: a 10-year review. Int J Dermatol. 2012;51(1):35-41.

9. Shornick JK, Bangert JL, Freeman RG, Gilliam JN. Herpes gestationis: clinical and histologic features of twenty-eight cases. J Am Acad Dermatol. 1983;8:214-224.

10. Kolodny RC. Herpes gentationis: a new assessment of incidence, diagnosis and fetal prognosis. Am J Obstet Gynecol. 1969;104:39-45.

11. Al-SaifF, Elisa A,Al-Homidy A,Al-Ageel A, Al-Mubarak M. Retrospective analysis of pemphigoid gestationis in 32 Saudi patients - clinicopathological features and a literature review. J Reprod Immunol. 2016;116:42-45.

12. Radia C, Salim G, Kawtar I, Zahra MF, Taoufiq H. Pemphigoid gestationis: a Moroccan study. Our Dermatol Online. 2017;8(2):128-132.

13. Shornick JK, Meek TJ, Nesbitt LT Jr, Gilliam JN. Herpes gestationis in blacks. Arch Dermatol. 1984;120(4):511-513.

14. Jenkins RE, Hern S, Black MM. Clinical features and management of 87 patients with pemphigoid gestationis. Clin Exp Dermatol. 1999;24:255-259.

15. Hallaji Z, Mortazavi H, Ashtari S, Nikoo A, Abdollahi M, Nasimia M. Pemphigoid gestationis: clinical and histologic features of twenty-three patients. Int J Womens Dermatol. 2017;3(2):86-90.

16. Mokni M, Fourati M, Karoui I, et al. Pemphigoid gestationis: a study of 20 cases. Ann Dermatol Venereol. 2004;131(11):953-956.

17. Soutou B, Aractingi S. Skin disease in pregnancy. Best Practice Res Clin Obstetr Gynaecol. 2015;29(5):732-740.

18. Lipozencic J, Ljubojevic S, Bukvic-Mokos Z. Pemphigoid gestationis. Clin Dermatol. 2012;30(1):51-55.

19. Vaughan Jones SA, Hern S, Nelson-Piercy C, Seed PT, Black MM. A prospective study of 200 women with dermatoses of pregnancy correlating clinical findings with hormonal and immunopathological profiles. Br J Dermatol. 1999;141:71-81.

20. Tani N, Kimura Y, Koga H, et al. Clinical and immunological profiles of 25 patients with pemphigoid gestationis. Br J Dermatol. 2014;172(1): 120-129.

21. Huilaja L, Mäkikallio K, Sormunen R, Lohi J, Hurskainen T, Tasanen K. Gestational pemphigoid: placental morphology and function. Acta Derm Venereol. 2013;93:33-38.

22. Holmes RC, Black M, Jurecka W, et al. Clues to the aetiology and pathogenesis of herpes gestationis. Br J Dermatol. 1983;109(2):131-139.

23. Patel F, Wilken R, Patel FB, et al. Pathophysiology of autoimmune bullous diseases: Nature versus nurture. Indian J Dermatol. 2017;62(3):262-267.

24. Borthwick GM, Sunderland CA, Holmes RC, Black MM, Stirrat GM. Abnormal expression of HLA-DR antigen in the placenta of a patient with pemphigoid gestationis. J Reprod Immunol. 1984;6:393-396.

25. Powell AM, Sakuma-Oyama Y, Oyama N, Black MM. Collagen XVII/ BP180: a collagenous transmembrane protein and component of the dermoepidermal anchoring complex. CED Clin Exp Dermatol. 2005;30(6):682-687.

26. Roth MM. Pregnancy dermatoses diagnosis, management, and controversies. Am J Clin Dermatol. 2011;12:25-41.

27. Shornick JK, Stastny P, Gilliam JN. High frequency of histocompatibility antigens HLA-DR3 and DR4 in herpes gestations. J Clin Invest. 1981;68:553-555.

28. Jenkins RE, Shornick J. Obstretic and Gynecologic Dermatology. 3rd ed. London: Elsevier; 2008.
29. Intong LR, Murrell DF. Pemphigoid gestationis: pathogenesis and clinical features. Dermatol Clin. 2011;29(3):447-452, ix.

30. Semkova K, Black M. Pemphigoid gestationis: current insights into pathogenesis and treatment. Eur J Obstet Gynecol Reprod Biol. 2009;145(2): 138-144.

31. Braun-Falco O, Plewig G, Wolff HH, Burgdorf WHC. Dermatology. Berlin Heidelberg: Springer; 2000.

32. Jenkins RE, Vaughan Jones S, Black MM. Conversion of pemphigoid gestationis to bullous pemphigoid--two refractory cases highlighting this association. Br J Dermatol. 1996;135(4):595-598.

33. Sitaru C, Powell J, Messer G, Bröcker EB, Wojnarowska F, Zillikens D. Immunoblotting and enzyme-linked immunosorbent assay for the diagnosis of pemphigoid gestationis. Obstet Gynecol. 2004;103(4):757-763.

34. Powell AM, Sakuma-Oyama Y, Oyama N, et al. Usefulness of BP180 NC16a enzyme-linked immunosorbent assay in the serodiagnosis of pemphigoid gestationis and in differentiating between pemphigoid gestationis and pruritic urticarial papules and plaques of pregnancy. Arch Dermatol. 2005;141(6):705-710.

35. Al Saif F, Jouen F, Hebert V, et al. Sensitivity and specificity of BP180 NC16A enzyme-linked immunosorbent assay for the diagnosis of pemphigoid gestationis. J Am Acad Dermatol. 2017;76(3):560-562.

36. Kwon EJ, Ntiamoah P, Shulman KJ. The utility of C4d immunohistochemistry on formalin-fixed paraffin-embedded tissue in the distinction of polymorphic eruption of pregnancy from pemphigoid gestationis. Am J Dermatopathol. 2013;35(8):787-791.

37. Black MM, Ambros-Rudolph C, Edwards L, Lynch PJ. Obstetric and Gynecologic Dermatology E-book. 3rd ed. London: Mosby; 2008.

38. Huilaja L, Mäkikallio K, Tasanen K. Gestational pemphigoid. Orphanet J Rare Dis. 2014;9:136.

39. Benediktsson R, Calder A, Edwards CR, Seckl JR. Placental 11 betahydroxysteroid dehydrogenase: a key regulator of fetal glucocorticoid exposure. Clin Endocrinol (Oxf). 1997;46(2):161-166.

40. Wöhrl S, Geusau A, Karlhofer F, Derfler K, Stingl G, Zillikens D. Pemphigoid gestationis: treatment with immunoapheresis. J Dtsch Dermatol Ges. 2003;1:126-130.

41. Westermann L, Hügel R, Meier M, et al. Glucocorticosteroid-resistant pemphigoid gestationis: successful treatment with adjuvant immunoadsorption. J Dermatol. 2012;39:168-171.

42. Carla dos Santos Rodrigues PF, del Mar Solana M, de Almeida LS, de Castro JC, Manuel, GM. Persistent herpes gestationis treated with high-dose intravenous immunoglobulin. Acta Dermatoven APA. 2007;87:184-186.

43. Doiron P, Pratt M. Antepartum intravenous immunoglobulin therapy in refractory pemphigoid gestationis: case report and literature review. J Cutan Med Surg. 2010;14(4):189-192.

44. Kreuter A, Harati A, Breuckmann F, Appelhans C, Altmeyer P. Intravenous immune globulin in the treatment of persistent pemphigoid gestationis. J Am Acad Dermatol. 2004;51(6):1027-1028.

45. Chi CC, Lee C, Wojnarowska F, Kirtschig G. Safety of topical corticosteroids in pregnancy. Cochrane Database Syst Rev. 2009;8(3):CD007346.

46. Götestam Skorpen C, Hoeltzenbein M, Tincani A, et al. The EULAR points to consider for use of antirheumatic drugs before pregnancy, and during pregnancy and lactation. Ann Rheum Dis. 2016;75(5):795-810.

47. Shornick JK, Black MM. Fetal risks in herpes gestationis. J Am Acad Dermatol. 1992;26:63-68.

48. Shornick JK, Black MM. Secondary autoimmune diseases in herpes gestationis (pemphigoid gestationis). JAm Acad Dermatol. 1992;26(4): 563-566. 


\section{Publish your work in this journal}

Clinical, Cosmetic and Investigational Dermatology is an international, peer-reviewed, open access, online journal that focuses on the latest clinical and experimental research in all aspects of skin disease and cosmetic interventions. This journal is included on PubMed. The manuscript management system is completely online and includes a very quick and fair peer-review system, which is all easy to use. Visit http://www.dovepress.com/testimonials.php to read real quotes from published authors

Submit your manuscript here: https://www.dovepress.com/clinical-cosmetic-and-investigational-dermatology-journal 